\title{
Depression in medical students: current insights
}

This article was published in the following Dove Press journal:

Advances in Medical Education and Practice

\section{Fiona Moir ${ }^{\prime}$ \\ jill Yielder ${ }^{2}$ \\ Jasmine Sanson ${ }^{3}$ \\ Yan Chen ${ }^{4}$}

'Department of General Practice and Primary Health Care, Faculty of Medical and Health Sciences, The University of Auckland, Auckland, New Zealand; ${ }^{2}$ Medical Programme Directorate, Faculty of Medical and Health Sciences, The University of Auckland, Auckland, New Zealand; ${ }^{3}$ Faculty of Medical and Health Sciences, The University of Auckland, Auckland, New Zealand; ${ }^{4}$ Centre for Medical and Health Sciences Education, Faculty of Medical and Health Sciences, The University of Auckland, Auckland, New Zealand
Correspondence: Fiona Moir Department of General Practice and Primary Healthcare, The University of Auckland, Private Bag 92019, Auckland I I 42, New Zealand

Tel +6493737599 ext84473

Fax +6493737948

Email f.moir@auckland.ac.nz

\begin{abstract}
Medical students are exposed to multiple factors during their academic and clinical study that have been shown to contribute to high levels of depression, anxiety, and stress. The purpose of this article was to explore the issue of depression in the medical student population, including prevalence, causes, and key issues, along with suggestions for early identification and support from one medical school in New Zealand. After establishing that the prevalence of depression is higher for medical students than the general population, the key issues explored include assessment used in the program, characteristics of the student population (such as Type A personality and perfectionism), resilience, selection procedures, students' motivation, and the nature of the clinical environment. This review includes several recommendations to improve students' psychological health such as positioning well-being within an overarching comprehensive workplace wellness model and integrating peer and faculty-led support into the day-to-day running of the institution. It also highlights the advantages of the addition of a well-being curriculum, as skills to prevent and manage distress and depression are relevant in supporting the competencies required by medical practitioners. It concludes that medical schools need wide-ranging strategies to address the complexities associated with the particular student population attracted to medicine and calls for educators to act, by noticing opportunities where they can introduce such initiatives into their medical programs.
\end{abstract}

Keywords: depression, well-being, medical students, medical school, mental health

\section{Introduction}

Can "medical student depression" be viewed as an opportunity as well as a problem? This statement does not intend in any way to minimize the severity of the issue, but invites us to reconsider that alongside managing risk we could also reflect on the opportunities that are offered by a medical school environment to prevent and manage depression in a high-risk group of young people, before they enter the workplace. Opening our minds to the possibilities that this stance might offer could enable us to view things from a slightly different perspective. Perhaps, the unique features of this student group and of the medical program itself could be explored with an eye for spotting "openings" where important "non-drug intervention" skills for depression could be learned and assimilated and stigmatizing attitudes transformed.

This review comments on the prevalence and causes of depression and other symptoms of psychological distress in medical students. It then explores some of the key issues that have been shown to contribute to high levels of depression, anxiety, and stress and concludes with recommendations for early identification and support. 


\section{Prevalence and causes of depression in medical students}

University students face various stressors such as academic requirements, time pressure and social adjustments, and medical students in particular, may face additional challenges such as the large workload, the time commitment and the number of assessments, as well as the pressures of a clinical environment. ${ }^{1}$ A recent meta-analysis showed that depression affects approximately one third of medical students worldwide, ${ }^{2}$ and it is also likely that the overall prevalence of depressive symptoms among medical students is higher than that reported in the general population. ${ }^{3}$ Students with depressive symptoms also suffer from other psychological difficulties, such as anxiety, burnout, suicidal thoughts, and substance abuse..$^{4-6}$ Research suggests that mental health deteriorates during medical school years and continues to decline when trainees enter the workforce. ${ }^{4}$ However, it has been shown that medical students can learn to adopt an active coping approach to deal with stress, which may act as a buffer to modulate their perceived stress levels. ${ }^{7}$ This is important, as students with lower stress levels are less likely to report suicidal thinking. ${ }^{8}$ A recent study showed that students are more likely to engage in active coping by their final year of medical school than they were in their earlier years. ${ }^{2}$ It is hard to discern if students learn these skills as they progress due to their medical school experiences, their training, or simply by maturing, but the evidence suggests that junior medical students are more at risk of suicidal thoughts or attempts. This underscores the importance of learning and applying healthy coping mechanisms early on in medical training. ${ }^{9}$ Research has shown that active coping strategies, such as positive framing, talking to family and friends, leisure activities, and exercising, can reduce the level of perceived stress among college students. ${ }^{10,11}$

The medical curriculum may contribute to the high prevalence of psychological ill-health among medical students. Dyrbye et $\mathrm{al}^{5}$ conducted a nationwide survey to compare the prevalence of burnout and other symptoms of psychological distress among medical students, residents, and early career physicians, relative to the general population. Among the medical professionals, being a medical student had the highest odds of depressive symptoms; medical students were also more likely to report depressive symptoms compared to college students of similar age from the general population. Even though certain aspects of mental health (eg, depressive symptoms) improve as students become residents and early career physicians, medical professionals are more likely to have depression and other psychological distress than do their counterparts across different stages of life. The prevalence of psychological distress was shown to be lower among a sample of students entering a medical program in the US than the age-matched sample in a general population. ${ }^{12}$ This sends a strong warning signal to medical educators that certain aspects of the medical curriculum may not be conducive to students maintaining healthy psychological states.

It is also important, when considering the literature reporting the prevalence of depression, to bear in mind the other confounding factors that may influence the picture. It may be, for example, that more support is currently provided for students with psychological difficulties than has been the case in the past, thereby influencing the likelihood of these students succeeding academically, ${ }^{13,14}$ remaining in their chosen course of study, ${ }^{15}$ and consequently being available to participate in a study. Furthermore, depression prevalence data are likely to be collected by self-complete questionnaires, which possess an inherent subjective bias. Which students choose to respond and how much they are prepared to disclose may also be affected by the level of stigma in the environment. It is therefore, reasonable to question whether increasing prevalence rates demonstrate a real increase in distress indicators or whether they are also reflecting changes in help-seeking behavior, support services, or reporting practices. ${ }^{16}$

\section{Key issues influencing medical students' well-being}

Given the high prevalence of psychological distress among medical students, medical educators, ideally, should have a good understanding of some of the key issues to be taken into consideration. These may contribute to the cause of the problems, or they may have the potential to influence what could be done to improve medical students' mental health. The key issues discussed in this review are the spectrum of well-being including help-seeking behavior and presentations of student distress, assessment, student motivation, and characteristics such as perfectionism and personality type, selection procedures, and the clinical environment.

\section{Well-being spectrum}

In 1946, the World Health Organization defined health as "a state of complete physical, mental and social well-being and not merely the absence of disease or infirmity", a definition clearly relevant today. ${ }^{17}$ It serves to remind us that there is a spectrum of well-being, with illness at one end and optimal well-being at the other, and that merely being "not depressed" is not the end goal. It would be beneficial for all people, 
including medical students, to be aware of their own place on the well-being spectrum at various times in their lives and to know what they might do to move up the spectrum.

The broad wellness spectrum highlights the fact that students are likely to be experiencing a wide range of psychological difficulties, many of which may overlap, such as anxiety and depression, and all of which could contribute to functional impairment. ${ }^{18}$ It is likely that there will be a high percentage of students attending medical school who have depression or a subthreshold level of distress. This raises the question about how important or not it might be to be aware of the likely "diagnoses" of medical students with psychological distress. Psychological conditions can sometimes be "diagnosed" retrospectively after suicide via psychological autopsy, so using a diagnostic label as a risk marker for serious harm may not be as useful as presumed for prediction, especially when these retrospectively derived figures regarding psychological illness as a risk factor for suicide can include diagnoses such as alcohol or substance abuse. ${ }^{19}$ While most doctors would argue that when it comes to assessing an individual patient, a diagnosis is important, as it influences decisions regarding management, others believe that categorization of distress is minimally helpful, medicalizes aspects of normal human suffering, and can potentially lead to overdiagnosis and unnecessary treatment. ${ }^{20} \mathrm{We}$ can extrapolate this to the medical school context to consider the fact that there may be a mixture of distress presentations. Students may have depression, stress, or burnout, which present in different ways. ${ }^{4}$ Some students could have a diagnosable mental health condition and could have an acute or chronic flare of depression, whereas others could be experiencing a short-term burst of distress without a background of psychological ill-health. It is worth reflecting on whether the same approach for both groups of students will work or not. The short-term distress group may not see themselves as "mentally ill" and may have more difficulty identifying with what is happening, even up to the point of suicidal ideation.

For example, see the following excerpt taken from a student portfolio, used as assessment of the Personal and Professional Skills (PPS) domain:

This last month has been very hard, in the sense that my mood has been repetitively crashing multiple times a day. There were four times where I just broke down, sometimes for a particular reason, others, there was no reason at all. It has been affecting me to the point where I can't, or won't, do anything because everything just seems too overwhelming. It's difficult to describe but it is almost as if I'm fighting to "keep my head above the water" most days. I've heard this phrase being thrown around by some of my friends who had experienced depression, but the idea of me having depression is just weird. I have everything to be grateful for, I live an amazing life, have a super supportive family and I love the career that I'm in. [Student 1]

Arguably, students who struggle with identifying themselves as being depressed may avoid or delay seeking help, ${ }^{21}$ which is one of the main barriers to care. The literature clearly shows that although some students seek professional help, many choose not to do so, due to commonly held perceptions about lack of confidentiality or perceptions of their symptoms as a failure or weakness. ${ }^{21}$ It seems sensible to enhance students' help-seeking behavior while they are at medical school if possible, as it is thought that poor help-seeking behavior is a likely cause of workplace accidents in doctors. ${ }^{22}$ This view is supported by research showing that depressed doctors have been shown to make six times as many medication errors as those who are not depressed. ${ }^{23}$

\section{Student motivation}

Given the intensity of medical training, it is important to consider additional factors that could influence student learning and well-being. Motivation is an important factor to help medical students maintain a healthy balance between study demand and personal well-being. Lyndon et $\mathrm{al}^{24}$ conducted a systematic review on medical student motivation and well-being. They found significant associations between medical student motivation (eg, intrinsic, extrinsic, "lack of motivation", self-efficacy, and self-regulation) and well-being, which was further moderated by demographic characteristics and curricular factors. Specifically, medical students who are more intrinsically motivated also experience better well-being. ${ }^{25}$ Motivation also contributes to the way students approach learning and to the outcome of study, such that highly motivated students also tend to perform better academically and adopt deep learning strategies. ${ }^{25}$

As mentioned previously, the extent to which motivation influences medical student well-being and learning could be moderated by curriculum variables. For instance, Henning et $\mathrm{al}^{26}$ found a positive relationship between preclinical medical students' self-reported motivation and their written academic achievement. In contrast, no such relationship was found between motivation and students' self-estimated clinical competence. It is critical for future research to continue to examine the different pathways that motivation contributes to student well-being and learning. 
An example of the interplay between motivation and psychological health can be seen in a Year 3 student's reflective diary entry:

[...] high levels of stress and low levels of motivation, confidence and self-esteem are rife among medical students, and I was no exception. Coming into my second year I found myself wondering whether I wanted to pursue medicine as a career, while being very interested in engineering, physics, maths and teaching. As a result, many of my experiences were tempered by this uncertainty, and I found it difficult to motivate myself to commit fully to my studies, and began to feel detached and lost. [Student 2]

In terms of motivating students to take steps toward improving their own psychological health to manage or prevent depression, it can be helpful to adhere to some of the principles from the health behavior literature. The spirit of motivational interviewing is composed of autonomy, collaboration, and evocation, ${ }^{27}$ and therefore it is important to encourage students to take ownership of the issue, to enhance their self-awareness, and to contemplate what improvements they might be prepared to make in their own health. This can be done through experiential exercises such as self-care diaries, well-being goals, or reflective writing as part of a portfolio. Spoon-feeding well-being information without engagement and "buy-in" from students is unlikely to be helpful. It is also critical to have staff on board for well-being initiatives aimed at addressing depression in students. Staff will need to know how to identify students in trouble, how to talk with those who are distressed, and where to refer students to should the need arise. They are also an integral part of any destigmatizing initiatives.

\section{Assessment}

There are multiple factors that seem to affect the depression and anxiety rates of medical students, as outlined by the authors earlier. One aspect included in the literature is the highly competitive environment of medical programs. While students entering the $\mathrm{MBChB}$ program have proven themselves to be high performing, academically capable, and motivated through their achievements before being accepted into the program, once they begin medical study, an assessment system that mathematically ranks students using a grading system from $\mathrm{D}$ to $\mathrm{A}+$ creates a perception of spread that may be artificial and is distressing for those students not achieving their previous " $A$ " scores. This may lead to coping strategies that are detrimental to emotional and physical well-being. According to Wilkinson, where [...]decisions about a test are driven primarily by mathematical methods [...] students will be ranked even if real differences in achievement are small". This ranking is at odds with a standards-based model of education that assumes that "all students can reach the required standard, albeit some taking longer than others. ${ }^{28}$

Several authors maintain that pass/fail grading promotes collaborative learning while de-emphasizing examination scores, reduces competition and anxiety, encourages cooperation among students, and enhances relations with teachers. ${ }^{4,29-31}$ Rohe et al ${ }^{30}$ cite Forsythe, who maintains that letter grades "transform intrinsically motivated learners into extrinsically motivated learners, precisely the opposite of currently espoused medical education principles". Dyrbye et $\mathrm{al},{ }^{4}$ in their study about sources of distress for medical students, stated that: "The A-F grading scheme, used to classify performance, often creates a competitive environment that promotes anxiety and peer competition rather than collaborative learning." Patel and David, in their study about prizes and distinctions that are the outcomes of a ranking system, noted that: "Rather than creating a stimulating and productive educational environment, rewards ultimately alienate students because they cause far more disappointment to those who tried but were unsuccessful than pleasure to the student who wins." 32 Furthermore, they argue that such a system engenders feelings of failure, self-criticism, and poor self-esteem in the majority of students, to the degree that they may not feel capable of undertaking tasks within their abilities. Instead of being encouraged to strive for success, they are forced to "adopt strategies (notably non-attendance) to avoid failure, public humiliation, and confirmation that they are less able than other students". 32

Bloodgood et $\mathrm{al}^{31}$ maintained that: "The principal attraction in moving toward a pass/fail grading system lies in the expectation that it will improve students' psychological well-being (reduce stress and anxiety), decrease competitiveness, and promote cooperative learning." These authors, along with Rohe et $\mathrm{al}^{30}$ and Robins et $\mathrm{al}^{29}$ carried out studies to measure the impact of the grading system on medical students. These three studies found that students graded using a pass-fail (or pass-borderline pass-fail variation) were significantly more satisfied with their evaluation and examination system and with the learning environment, exhibited a significant increase in well-being, had greater satisfaction with their personal lives, and perceived less stress and greater group cohesion. Furthermore, they found that student motivation to achieve excellence remained intact and that student performance (in courses, clerkships, licensing 
examinations, and residency placements) and attendance did not decline. ${ }^{29-31}$

In the MBChB program at the University of Auckland, Years 1-3 use the standard university 11-point grading scale, while the final 3 years are graded as pass, fail, or distinction. Moves to change this have not been successful in the early years of the program. However, the introduction of progress testing and year-long courses that allow for longitudinal assessment across multiple assessment points have been implemented to reduce the high-stakes nature of previous assessment methods.

\section{Characteristics of students}

The desire to be excellent coupled with a competitive learning environment engages the classic "Type A" medical students in learning behavior that may be detrimental to psychological health. Type A behavior pattern is defined by Lohse et $\mathrm{al}^{33}$ as an action-emotion complex, with individuals characterized by ambition, display of highly competitive attitudes toward achievement, and feeling compelled to "work harder than Type B individuals to accomplish tasks, regardless of external stressors". ${ }^{34}$ They are said to have an exaggerated sense of urgency with regard to time, preferring to spend it on things they deem as priorities, and possibly becoming aggressive, hostile, or impatient in frustrating situations. ${ }^{34}$ These authors suggest that Type A characteristics begin with a predisposition for competitiveness and then lead to the manifestation of characteristics such as ambitiousness, aggressiveness, impatience, and physiological behaviors such as muscle tension, a hurried pace, and alertness.

While anecdotally it is recognized that medical students exhibit Type A characteristics, there appears to be little evidence to support this, although a study by Alfulaij and Alnasir ${ }^{35}$ found that of 77 Year 1 medical students in Bahrain in $2013,76.6 \%$ had Type A personality, as opposed to $23.4 \%$ with Type B. They also found that Type A personality was more prevalent among female medical students $(63.6 \%$ as compared to $36.4 \%$ ). These authors linked Type A personality with endeavor for perfectionism. While the drive exhibited by this personality type may result in extraordinary achievements, pressure to achieve may result in inappropriate selfexpectations and psychologically related stress disorders such as anxiety and depression. Jackson, ${ }^{6}$ in the study about the perfectionist tendencies of dentists, maintained that an inability to settle for "good enough" results in inflexible rigidity and limited the person to only the two options of perfection or failure. This may create an untenable long-term work pressure. Alfulaij and Alnasir ${ }^{35}$ commented that Type A personality doctors may fail to schedule time for relaxation and, since they have been shown to have a more internalized locus of control, they may internalize the burden related to their patients. They recommended that medical students are educated about the implications of Type A behavior early in their program, including ways of improving their psychological health. This recommendation is in accord with the introduction of the Health and Well-being theme within the PPS domain in the Auckland medical program, although Type A personality has not explicitly been introduced as a topic within the domain.

Students have described the link between perfectionism and depression in their portfolios, for example:

\section{My mother asked me what was wrong. I said "nothing." I felt I had to maintain a veneer of strength, I saw anxiety as a flaw, and in front of my parents I had to maintain this image of perfection because this is what I thought they expected of me, that is what they pride themselves on - a perfect child. [Student 3]}

In the context of the notion that many doctors are attracted to the profession through their own experiences of physical or psychological suffering, psychological health and resilience need to be considered at the entry point to medical program in both their positive and negative aspects. The notion of the "wounded healer" is referred to by Jackson as "[...] the inner 'woundedness' of a healer - the healer's own suffering and vulnerability, which have been said to contribute crucially to the capacity to heal". ${ }^{36}$ Jackson $^{36}$ is referring to the way a person's experience of illness, when worked through in a meaningful way, may result in attitudes and sensitivities that enhance the capacity to work empathically with others. This is acknowledged as a significant factor in both vocational choice and in contribution to healing the patient, particularly in many of the helping professions, such as psychotherapy, psychiatry, and professions that involve counseling and pastoral care. As such, it is important to consider the motivations of students in applying to study medicine - if they identify past illness, this could be considered beneficial if this has led to a deepening of understanding, while at the same time, it is important to establish current levels of health and likely future resilience.

\section{Selection process}

Recent efforts to quantify characteristics that establish "higher or lower risk" for psychological difficulties in medical students have led to the suggestion by some authors that more resilient students could be chosen at the point of medical school selection. ${ }^{37}$ However, there are multiple factors to be taken into consideration with selection procedures, such as 
equity admission pathways, to ensure that a broad range of students are selected to reflect cultural and economic diversity and potentially reduce health inequities. Students selected via these pathways may bring with them levels of complexity, including pastoral and socioeconomic issues, ${ }^{38}$ factors that may impact on their psychological health. Similarly, graduate-entry students and undergraduate students may have different coping mechanisms, which may also influence their psychological health. ${ }^{39}$

Tyssen et $\mathrm{al}^{40}$ found the highest risk group for deterioration in psychological health during medical school was formed by those students with high conscientiousness and high neuroticism, while the group most protected from worsening psychological health was formed from those with high extroversion, low neuroticism, and low conscientiousness. However, conscientiousness is the trait most significantly associated with better medical school performance. ${ }^{41}$ Therefore, it could be argued that selecting students at low risk of psychological ill-health at medical school entry does not necessarily lead to the selection of those with the characteristics considered best for a doctor to possess (eg, empathy formed through personal experience). Instead, the focus might be better placed on reducing the elements of medical school that contribute to psychological distress. In fact, it could be suggested that this is simply an extension to the victim-blaming culture that creates psychological distress within the health care system. Certainly, doctors do need a certain level of resilience and psychological stability to be able to capably operate while under pressure, make critical decisions, problem solve, and communicate clearly. They also need to be able to withstand the demands of an emotionally and sometimes physically demanding job and handle responsibility, uncertainty, and heavy workloads. However, there is a balance to be found that encompasses some elements of selecting for more resilient students without this being at the expense of selecting for the best doctors. In addition, Faculty and the profession could also look at what might be done to reduce some of these demands and to tackle the flaws in the health care system.

\section{Clinical environment}

In the last few years, there has been an increased focus on the issue of bullying and harassment of medical trainees in New Zealand and internationally. ${ }^{42-44}$ It is clear that this practice is widespread and can have a dramatic impact on students' psychological health and ability to learn. ${ }^{45}$ In one study, approximately one-sixth of study participants stated that a bullying or harassment experience had made them consider leaving medical school. ${ }^{45}$ The Medical Council of New Zealand and the Royal Australasian College of Surgeons have shown leadership in beginning to tackle this issue. The World Medical Association has recently adopted a policy statement put forward by The New Zealand Medical Association and has issued a position statement condemning bullying and harassment and stating that international action is required. ${ }^{46}$ In order to tackle this problem, it will take a whole system approach that aims to influence the culture at all levels for bystanders, perpetrators, and victims and includes changes in reporting systems, medical training, and awareness raising. ${ }^{42}$ Positive role modeling by all clinicians is key, as the hidden curriculum strongly influences students' personal and professional development ${ }^{47,48}$ to enable a change in culture away from intimidation and toward collegiality and the acceptance of vulnerability. This could create a different sort of environment, where students are not fearful of admitting ignorance or distress, and these are viewed as a normal part of growth and learning, not as a weakness.

For example, a student expressed his/her views about medical students' mental health in his/her reflective diary:

[...] as medical students, we are so out of touch sometimes.

We can't really share our concerns or worries because we have to appear to be at our best all the time. There is this underlying pressure to be perfect. And there is no way that can be conducive to good mental health I reckon [...]. [Student 4]

\section{Recommendations Strategic framework}

We believe that improving rates and levels of depression in medical students requires a comprehensive approach and that addressing specific aspects of this issue in isolation is less likely to be successful. Overarching guidelines for tertiary institutions have been developed to enable organizations to pinpoint strategic gaps in their systems. ${ }^{49}$ Models have been specifically developed for medical schools, such as the "three pillars" from The Vanderbilt School of Medicine, which recommends the implementation of a well-being curriculum, student-led support, and faculty services. ${ }^{50}$ Another good example of a strategic support framework developed for medical students is the Four-Tier Continuum of Academic and Behavioural Support integrated model from the Netherlands. This model targets students at all levels and emphasizes the importance of the provision of academic and behavioral support and evidence-based early intervention. ${ }^{51}$ 


\section{Well-being curriculum}

There are strong arguments for the inclusion of well-being in a medical curriculum, such as the impact of doctors' personal health practices on their communication and patient care. ${ }^{23,52,53}$ Improving doctors' well-being also has been shown to enhance their empathy, communication skills, and reflective practice. ${ }^{54}$ In 2014, when The Medical Council of New Zealand introduced the New Zealand Curriculum Framework for newly graduated doctors, "Personal Well-being" was part of it. ${ }^{55}$ This section of the framework included learning objectives such as "balance availability to others with care for personal health, managing fatigue, stress and illness" and emphasized that it was important for doctors to have their own general practitioner. ${ }^{56}$

There is a wide range of possible content that could be included in a well-being curriculum for medical students, and developers need to choose carefully as they are likely to be "competing" with other disciplines for space in the curriculum. Content can also be assessed if required, bearing in mind that for many students, assessment drives learning. Topics such as sleep, exercise, problem-solving, and an ability to manage stress and worried thinking can be taught, and many of these skills are the first "step" of non-drug interventions for managing depression. ${ }^{57}$ Active coping is a key skill to be included, as passive coping mechanisms have been linked to poorer long-term psychological health. ${ }^{58}$ It may also be possible to target specific personality traits that can be common in this student group. For example, optional sessions could be included to assist students with managing perfectionist tendencies, such as the opportunity to learn selfcompassion exercises. This could enable students to be able to optimize the benefits of perfectionism while minimizing the detriments.

When considering topics to include in a health and wellbeing curriculum, it may be worth exploring whether there are unique "medical student or medical school factors" that lend themselves to learning useful skills for preventing and managing depression. To have gained successful entry to medical school, students in the program will be academically capable of assimilating a relevant knowledge base. It is also likely that they will be motivated to succeed, which may inspire them to assimilate skills that might enable them to be more "successful". For example, increased emotional intelligence has been cited as a leadership quality ${ }^{59}$ which may be attractive to some students, while at the same time, self-awareness and self-management can be useful stress management skills that may prevent a depressive spiral. ${ }^{60}$ Mindfulness, a state of mind where one chooses to focus one's mind on the present moment in a non-judgmental manner, is a good example of a topic that can be included as a useful component of medical training on both a professional and a personal level. The ability to step back, tune out distractions, and choose what to pay attention to is clearly a skill that will be useful for a doctor, for instance, if they are required to focus on carrying out a procedure in a high-stress situation, where it can function as a "technique" and on the spot destressor. Mindfulness meditation training can also improve focus, productivity, and effective learning skills, and for some, it is a spiritual practice and way of being. In addition, mindfulness has also been shown to prevent the recurrence of depression. ${ }^{57}$

The core components of the University of Auckland SAFE-DRS curriculum are "Self-Care Skills", "Accessing Help", "Focused Attention", "Emotional Intelligence", "Doctor as Patient and Colleague", "Reflective Practice", and "Stress Resistance". There are some practical lessons to be learnt in developing and implementing a health and well-being curriculum in a medical program, such as the importance of referring to available evidence to impress upon others the significance of well-being initiatives. Although the recent New Zealand Health and Safety at Work Act $2015^{61}$ may assist in this regard in the future, there may be some people who will not see "well-being" as a priority or who will brush it off as being "fluffy" or optional. The best position to shift this mind-set is by clearly and consistently drawing links to service quality and safety and by encouraging autonomy and collaboration, integrating others' ideas whenever possible. While there needs to be governance to set up systems, an exclusively top-down approach is unlikely to be as successful. Well-being is "personal" and is seen by many people as a sensitive topic. There is a range of views on where the boundary lies between peoples' personal and professional lives, and this needs to be taken into consideration.

A well-being curriculum is the fence at the top of the cliff, not the ambulance at the bottom. In searching for better metaphors, perhaps in time we can hope that students will move away from the cliff edge altogether and turn instead to face the mountain of possibilities, thriving on challenge and flourishing. There is a spectrum of well-being, and being "not depressed" is only the midpoint, not the top end of the scale.

\section{Peer-led initiatives}

Student-led approaches may include multiple types of wellbeing activities, for instance, social and supportive networking activities. This is important, as strong social ties are a protective factor for depression, ${ }^{62}$ and being connected to 
other people is a basic human need that impacts on wellbeing. One of the key recommendations of The Youth Development Strategy Aotearoa is to involve young people in a meaningful way in both the development and the delivery of new initiatives. ${ }^{63}$ There is also evidence to show that medical students prefer to approach peers for support rather than seeking help from health professionals or faculty members. ${ }^{64}$ In the extreme situation of suicide, suicidal young adults prefer to talk to a peer rather than a parent, a staff member, or a counsellor. ${ }^{65}$ One New Zealand study showed that students were willing to seek help for a peer who expressed suicidal concerns, while often being less willing to seek help for themselves. ${ }^{66}$ Peer-led interventions have been shown to be feasible and acceptable, ${ }^{67}$ for example, the Oxford Peer Support program, which has been run successfully out of the counseling service at Oxford University for over 20 years. ${ }^{68}$ Peer leader training will need to be provided, as students may feel that they do not have the skills or resources to provide this support. ${ }^{69}$ In the USA, peer support programs in medical schools have been shown to often go hand in hand with peer teaching, ${ }^{70}$ which is another possibility to be considered. Peer support programs do not have to be face-to-face; the Internet could be another medium to deliver support programs as Shaw and Gant ${ }^{71}$ showed that participants reported significant decreases in loneliness and depression after five chat sessions with an anonymous partner online.

For example, the following excerpt from a peer leader illustrates that there are benefits for the leaders as well as the students they support:

Not sure how psychologists and counsellors do this for a living. It's exhausting listening to someone share their concerns, feelings, crises, and I think in particular because I still haven't mastered the art of not feeling for them, as if I'm taking on board their feelings as my own. I do feel like I have helped her in a way. Like, it was good to see her walk away looking that bit lighter and that bit more purposeful and determined. But extending from that, I think it also helped me as well. Having that human connection, that interaction, made me feel in touch and made me feel human. [Student 5]

\section{Faculty-led initiatives}

In terms of preventing and managing depression, early identification and management are vital and encompass independent university services providing support to students and some aligned faculty support. One possibility is to employ an independent person in a student support role, who is ideally not also involved in assessment, as it is possible that a dual role can result in a conflict of interest and unhelpful power dynamics. When considering the provision of student support, there are several key issues such as giving students a choice of services and people to approach, along with transparency regarding roles and documentation, flexible services, and practicalities such as cost and location.

Anecdotally, some students have expressed a belief that perhaps Faculty view students with psychological difficulties as a process of "natural selection" and have no desire or impetus to help those struggling through the program, perhaps preferring that those students would leave medical school as they appear to not be suited to the environment. Although this view is at odds with the many initiatives that have been put in place in medical schools worldwide, such as well-being training and support services, ${ }^{72}$ if this perception persists, it could enable stigma to flourish and have a negative impact on students' help-seeking behavior. It could be helpful to dispel this belief by providing information about periods during the year where it may be appropriate to take some additional time off for illhealth if required, with faculty approval, so that students may feel they can "hold on" until this point. It is also important to have appropriate deferral policies in place, so that students can take extended time, such as a year or even two, away from the program, without negative implications for their career. Many students appear to believe that anything that jeopardizes their progression through the program is undesirable and that this is to be avoided, regardless of the severity of psychological deterioration or the misery they may be experiencing.

\section{Conclusion}

Depression in medical students is a complex issue, compounded by many factors including selection procedures, the likely personalities of those attracted to medicine, assessment methods, and the clinical environment. Students' motivation to learn and their willingness and ability to take ownership and manage their own health also play a part. While certain groups of students may be more at risk of becoming unwell, ${ }^{73}$ students are likely to experience a broad range of psychological difficulties across the whole well-being spectrum, and therefore medical schools will need wide-ranging strategies to assist with the different concerns and levels of distress. It is clear that many of the skills that may be helpful in combatting depression and preventing its recurrence will also be useful competencies for a medical practitioner, as well as having clear links to patient safety and quality of care, and this provides a rationale for their inclusion in the curriculum. Additionally, it could be useful to strengthen support provided 
by the faculty and the students and to normalize "well-being" to combat stigma. Ideally, wellness initiatives should form part of a comprehensive model, be core business for all staff and students, and be integrated into the values and day-to-day operation of the organization.

If what we currently have is a medical culture rife with exhaustion, learned helplessness, bullying, and psychological distress, we need to persevere until we discover how to improve it. The recent addition to the Hippocratic Oath ${ }^{74}$ of doctors attending to their own health is encouraging. ${ }^{75}$ Perhaps, it is not an unrealistic aspiration to aim for a culture which focuses on practitioner well-being, self-compassion and collegiality and strive for this to become "the new normal" for our next generation of doctors. By adopting a proactive, strength-based approach to wellness, which involves meaningful participation by students and staff, we might be able to see an improvement in the prevalence of depression in medical students. There might be advantages in viewing this issue not just as a risk to be managed or a problem to be solved, but as a clear message that things need to change in the profession. As well as being centers of teaching and learning, medical programs are spheres of influence. They are places where students develop their strategies, attitudes, and thinking and have multiple opportunities to engage, inspire, and role model a new way of being, both in self-care and in professional behaviors. Medical schools could hold the key to effect change and may be in the best position to enable medical students, educators, and clinicians to work together to build a healthier workplace culture.

\section{Disclosure}

The authors report no conflicts of interest in this work.

\section{References}

1. Sreeramareddy CT, Shankar PR, Binu VS, Mukhopadhyay C, Ray B, Menezes RG. Psychological morbidity, sources of stress and coping strategies among undergraduate medical students of Nepal. BMC Med Educ. 2007;7:26.

2. Puthran R, Zhang MW, Tam WW, Ho RC. Prevalence of depression amongst medical students: a meta-analysis. Med Educ. 2016;50(4): 456-468.

3. Rotenstein LS, Ramos MA, Torre M, et al. Prevalence of depression, depressive symptoms, and suicidal ideation among medical students: a systematic review and meta-analysis. JAMA. 2016;316(21): 2214-2236.

4. Dyrbye LN, Thomas MR, Shanafelt TD. Medical student distress: causes, consequences, and proposed solutions. Mayo Clin Proc. 2005;80(12): 1613-1622.

5. Dyrbye LN, West CP, Satele D, et al. Burnout among U.S. medical students, residents, and early career physicians relative to the general U.S. population. Acad Med. 2014;89(3):443-451.

6. Jackson W. Mindfulness and perfectionism in dentistry. J Mass Dent Soc. 2017;66(1):12-13.

7. Heinen I, Bullinger M, Kocalevent RD. Perceived stress in first year medical students - associations with personal resources and emotional distress. BMC Med Educ. 2017;17(1):4.
8. Rosiek A, Rosiek-Kryszewska A, Leksowski L, Leksowski K. Chronic stress and suicidal thinking among medical students. Int J Environ Res Public Health. 2016;13(2):212.

9. Miletic V, Lukovic JA, Ratkovic N, Aleksic D, Grgurevic A. Demographic risk factors for suicide and depression among Serbian medical school students. Soc Psychiatry Psychiatr Epidemiol. 2015;50(4):633-638.

10. Pierceall EA, Keim MC. Stress and coping strategies among community college students. Community Coll J Res Pract. 2007;31(9):703-712.

11. Bamuhair SS, Al Farhan AI, Althubaiti A, Agha S, Rahman S, Ibrahim N. Sources of stress and coping strategies among undergraduate medical students enrolled in a problem-based learning curriculum. J Biomed Educ. 2015;2015:8.

12. Brazeau CM, Shanafelt T, Durning SJ, et al. Distress among matriculating medical students relative to the general population. Acad Med. 2014;89(11):1520-1525.

13. Stallman HM. Psychological distress in university students: a comparison with general population data. Aust Psychol. 2010;45(4):249-257.

14. Gortner E-M. The Mental and Physical Well-Being of Formerly Depressed College Students: A Preventive Intervention Study. Texas: University of Texas at Austin; 2006.

15. Dyrbye LN, Thomas MR, Power DV, et al. Burnout and serious thoughts of dropping out of medical school: a multi-institutional study. Acad Med. 2010;85(1):94-102.

16. Hunt J, Eisenberg D. Mental health problems and help-seeking behaviour among college students. J Adolesc Health. 2010;46(1):3-10.

17. Piko B. Teaching the mental and social aspects of medicine in Eastern Europe: role of the WHO definition of health. Adm Policy Ment Health. 1999;26(6):435-438.

18. Löwe B, Spitzer RL, Williams JB, Mussell M, Schellberg D, Kroenke K. Depression, anxiety and somatization in primary care: syndrome overlap and functional impairment. Gen Hosp Psychiatry. 2008;30(3):191-199.

19. Conwell Y, Duberstein PR, Cox C, Herrmann JH, Forbes NT, Caine ED. Relationships of age and axis I diagnoses in victims of completed suicide: a psychological autopsy study. Am J Psychiatry. 1996;153(8): 1001-1008.

20. Dowrick C, Frances A. Medicalising unhappiness: new classification of depression risks more patients being put on drug treatment from which they will not benefit. BMJ. 2013;347:f7140.

21. Givens JL, Tjia J. Depressed medical students' use of mental health services and barriers to use. Acad Med. 2002;77(9):918-921.

22. Firth-Cozens J. Interventions to improve physicians' well-being and patient care. Soc Sci Med. 2001;52(2):215-222.

23. Fahrenkopf AM, Sectish TC, Barger LK, et al. Rates of medication errors among depressed and burnt out residents: prospective cohort study. BMJ. 2008;336(7642):488.

24. Lyndon MP, Strom JM, Alyami HM, et al. The relationship between academic assessment and psychological distress among medical students: a systematic review. Perspect Med Educ. 2014;3(6):405-418.

25. Kusurkar RA, Croiset G, Galindo-Garré F, Ten Cate O. Motivational profiles of medical students: association with study effort, academic performance and exhaustion. BMC Med Educ. 2013;13(1):87.

26. Henning MA, Krägeloh CU, Hawken SJ, Doherty I, Zhao Y, Shulruf B. Motivation to learn, quality of life and estimated academic achievement: medical students studying in New Zealand. Med Sci Educ. 2011;21(2):142-150.

27. Rollnick S, Miller WR. What is motivational interviewing? Behav Cogn Psychother. 1995;23(4):325-334.

28. Wilkinson T. The Educational Impact of a Change to Standards-Based Assessments within a Medical Curriculum. Dunedin: University of Otago; 2005.

29. Robins LS, Fantone JC, Oh MS, Alexander GL, Shlafer M, Davis WK. The effect of pass/fail grading and weekly quizzes on first-year students' performances and satisfaction. Acad Med. 1995;70(4):327-329.

30. Rohe DE, Barrier PA, Clark MM, Cook DA, Vickers KS, Decker PA. The benefits of pass-fail grading on stress, mood, and group cohesion in medical students. Paper Presented at Mayo Clinic Proceedings. Rochester, UK; 2006. 
31. Bloodgood RA, Short JG, Jackson JM, Martindale JR. A change to pass/fail grading in the first two years at one medical school results in improved psychological well-being. Acad Med. 2009;84(5):655-662.

32. Patel L, David T. Everybody has won, and all must have prizes. Lancet. 1996;348(9040):1497.

33. Lohse T, Rohrmann S, Richard A, Bopp M, Faeh D; Swiss National Cohort Study Group. Type A personality and mortality: competitiveness but not speed is associated with increased risk. Atherosclerosis. 2017;262:19-24.

34. Watson WE, Minzenmayer T, Bowler M. Type A personality characteristics and the effect on individual and team academic performance. J Appl Soc Psychol. 2006;36(5):1110-1128.

35. Alfulaij AR, Alnasir FA. Type A and B personalities from a psychological perspective among medical students. Psychol Res. 2014;4(7):559-566.

36. Jackson SW. Presidential address: the wounded healer. Bull Hist Med. 2001;75(1):1-36.

37. Powis D. Selecting medical students: an unresolved challenge. Med Teach. 2015;37(3):252-260.

38. Curtis E, Wikaire E, Jiang Y, et al. Examining the predictors of academic outcomes for indigenous Maori, Pacific and rural students admitted into medicine via two equity pathways: a retrospective observational study at the University of Auckland, Aotearoa New Zealand. BMJ Open. 2017;7(8):e017276.

39. Zvauya R, Oyebode F, Day EJ, Thomas CP, Jones LA. A comparison of stress levels, coping styles and psychological morbidity between graduate-entry and traditional undergraduate medical students during the first 2 years at a UK medical school. BMC Res Notes. 2017;10(1):93.

40. Tyssen R, Dolatowski FC, Rovik JO, et al. Personality traits and types predict medical school stress: a six-year longitudinal and nationwide study. Med Educ. 2007;41(8):781-787.

41. Doherty EM, Nugent E. Personality factors and medical training: a review of the literature. Med Educ. 2011;45(2):132-140.

42. Crampton P, Wilkinson T, Anderson L, Walthert S, Wilson H. Bullying in health care settings: time for a whole-of-system response. $N Z$ Med $J$. 2015;128(1424):10-13.

43. Meo SA, Usmani AM. Bullying of medical students. J Coll Physicians Surg Pak. 2011;21(9):579; author reply 579.

44. Mukhtar F, Daud S, Manzoor I, et al. Bullying of medical students. J Coll Physicians Surg Pak. 2010;20(12):814-818.

45. Wilkinson TJ, Gill DJ, Fitzjohn J, Palmer CL, Mulder RT. The impact on students of adverse experiences during medical school. Med Teach. 2006;28(2):129-135.

46. World Medical Association [webpage on the Internet]. WMA Statement on Bullying and Harrassment within the Profession. Available from: https://www.wma.net/policies-post/wma-statement-on-bullying-andharassment-within-the-profession/. Accessed November 15, 2017.

47. Hopkins L, Saciragic L, Kim J, Posner G. The hidden curriculum: exposing the unintended lessons of medical education. Cureus. 2016;8(10):e845.

48. Bandini J, Mitchell C, Epstein-Peterson ZD, et al. Student and faculty reflections of the hidden curriculum. Am J Hosp Palliat Care. 2017;34(1):57-63.

49. Guidelines for Tertiary Education Institutions to Facilitate Improved Educational Outcomes for Students with a Mental Illness. Melbourne: Orygen Youth Health Research Centre, University of Melbourne; 2011.

50. Drolet BC, Rodgers S. A comprehensive medical student wellness program - design and implementation at Vanderbilt School of Medicine. Acad Med. 2010;85(1):103-110.

51. Stegers-Jager KM, Cohen-Schotanus J, Themmen APN. The four-tier continuum of academic and behavioral support (4T-CABS) model: an integrated model for medical student success. Acad Med. 2017;92(11): $1525-1530$.

52. Frank E, Galuska DA, Elon LK, Wright EH. Personal and clinical exercise-related attitudes and behaviors of freshmen U.S. medical students. Res Q Exerc Sport. 2004;75(2):112-121.

53. Frank E. Physician health and patient care. JAMA. 2004;291(5):637.
54. Neumann M, Edelhauser F, Tauschel D, et al. Empathy decline and its reasons: a systematic review of studies with medical students and residents. Acad Med. 2011;86(8):996-1009.

55. Medical Council of New Zealand. New Zealand Curriculum Framework for Prevocational Medical Training. 2014. Available from: https:// www.mcnz.org.nz/assets/News-and-Publications/NZCF26Feb2014. pdf. Accessed March 15, 2018

56. Henning MA, Hawken SJ, Hill AG. The quality of life of New Zealand doctors and medical students: what can be done to avoid burnout? $N Z$ Med J. 2009;122(1307):102-110.

57. (NICE) TNIfHaCE [webpage on the Internet]. Quality Statement 13: Residual Symptoms or Risk of Relapse. 2011. Available from: https:// www.nice.org.uk/guidance/qs8/chapter/Quality-statement-13-Residualsymptoms-or-risk-of-relapse. Accessed November 15, 2017.

58. Tyssen R, Vaglum P, Gronvold NT, Ekeberg O. Factors in medical school that predict postgraduate mental health problems in need of treatment. A nationwide and longitudinal study. Med Educ. 2001;35(2):110-120.

59. Hariharan M, Padhy M. Emotional intelligence of doctors. Soc Sci Int. 2017;27(1):15-22.

60. Arora S, Ashrafian H, Davis R, Athanasiou T, Darzi A, Sevdalis N. Emotional intelligence in medicine: a systematic review through the context of the ACGME competencies. Med Educ. 2010;44(8): $749-764$.

61. Government NZ. Health and Safety at Work Act 2015. 2015. Available from: http://www.legislation.govt.nz/act/public/2015/0070/latest/ DLM5976660.html. Accessed March 15, 2018.

62. Ball S, Bax A. Self-care in medical education: effectiveness of health-habits interventions for first-year medical students. Acad Med. 2002;77(9):911-917.

63. Ministry of Youth Affairs. Youth Development Strategy Aotearoa: Action for Child and Youth Development. Wellington: New Zealand Ministry of Youth Affairs; 2002.

64. Huggard PK. Can I cope with what I have to do? Where do I go for help? Poster Presented at Health Care 2005: Emerging Issues. Pocatello, Idaho; 2005.

65. Kalafat J, Elias M. Adolescents' experience with and response to suicidal peers. Suicide Life Threat Behav. 1992;22(3):315-321.

66. Curtis C. Youth perceptions of suicide and help-seeking: 'they'd think I was weak or "mental”, J Youth Stud. 2010;13(6):699-715.

67. Moir F, Henning M, Hassed C, Moyes SA, Elley CR. A peer-support and mindfulness program to improve the mental health of medical students. Teach Learn Med. 2016;28(3):293-302.

68. Ford A. Peer Support in Colleges and Universities. 2nd ed. Oxford: Oxford University Counselling Service; 2004.

69. Reavley N, Jorm AF. Prevention and early intervention to improve mental health in higher education students: a review. Early Interv Psychiatry. 2010;4(2):132-142.

70. Moore-West M, Hennessy SA, Meilman PW, O'Donnell JF. The presence of student-based peer advising, peer tutoring, and performance evaluation programs among U.S. medical schools. Acad Med. 1990;65(10):660-661.

71. Shaw LH, Gant LM. In defense of the Internet: the relationship between Internet communication and depression, loneliness, self-esteem, and perceived social support. Cyberpsychol Behav. 2004;5(2):157-171.

72. Cardiff University. Medic Support. Available from: http://medicapps. cardiff.ac.uk/medicsupport/documents/medic_support_info_sheet_ oct_2015.pdf. Accessed November 15, 2017.

73. Henning M, Krägeloh C, Fiona M, Doherty I, Hawken S. Quality of life: international and domestic students studying medicine in New Zealand. Perspect Med Educ. 2012;1(3):13.

74. Miles SH. The Hippocratic Oath and the Ethics of Medicine. Oxford, New York: Oxford University Press; 2004.

75. Radio New Zealand [webpage on the Internet]. NZ Doctor's Hippocratic Oath Change Passed. Available from: http://www.radionz.co.nz/news/ world/341592/nz-doctor-s-hippocratic-oath-change-passed. Accessed November 15, 2017. 
Advances in Medical Education and Practice

Dovepress

\section{Publish your work in this journal}

Advances in Medical Education and Practice is an international, peerreviewed, open access journal that aims to present and publish research on Medical Education covering medical, dental, nursing and allied health care professional education. The journal covers undergraduate education, postgraduate training and continuing medical education including emerging trends and innovative models linking education, research, and health care services. The manuscript management system is completely online and includes a very quick and fair peer-review system. Visit http://www.dovepress.com/testimonials.php to read real quotes from published authors.

Submit your manuscript here: http://www.dovepress.com/advances-in-medical-education-and-practice-journal 